\title{
Vom „Back Channel“ zum „Track 1,5“ - Begegnungen mit Egon Bahr im deutsch-deutschen Sicherheitsdialog
}

\author{
Hans J. Giessmann
}

\begin{abstract}
The experience of working together with Egon Bahr began for Hans J. Giessmann as staff member of the Institute for International Politics in East Berlin. The following article reflects on the contribution of the German-German security policy dialogue, and related joint research consultations, in particular, to pave the way for a peaceful regime change in East Germany and the following re-unification of Germany. Egon Bahr's role in that was pivotal. His approach of "change through rapprochement" helped erode the legitimacy of having two German states in Europe. In the following, the development from the "Back Channel" to "Track 1.5", which was driven by Bahr's relentless effort to establish a common dialogue between East and West Germany, is illustrated.
\end{abstract}

Keywords: German Unity, change through rapprochement, Back Channel, Track 1.5, security dialogue Stichworte: Deutsche Einheit, Wandel durch Annäherung, Back Channel, Track 1,5, Sicherheitsdialog

\section{Einleitung}

$\mathrm{E}$ gon Bahr traf ich das erste Mal 1985 durch einen überaus glücklichen Zufall. Während eines kurzfristig vereinbarten Gesprächs mit seinem persönlichen Referenten, Uwe Stehr, öffnete sich plötzlich die Tür, er steckte den Kopf herein und war sichtlich ungehalten, weil offenbar Dringendes mit seinem Mitarbeiter zu besprechen war. Als er hörte, woher ich kam - zur damaligen Zeit war ich wissenschaftlicher Mitarbeiter am Institut für Internationale Politik und Wirtschaft (IPW) in Ost-Berlin, und nahm während einer meiner ersten sogenannten „Westreisen“ an einer Konferenz in Bonn teil - veränderte sich seine Miene sofort. Die Dringlichkeit seines Anliegens schien vergessen. Mit den Worten: „Das ist ja fabelhaft", betrat er das winzige Büro seines Mitarbeiters, nahm Platz und verwickelte mich sogleich ins Gespräch. Bahr war gerade erst von Konsultationen aus Moskau zurückgekehrt, tief beeindruckt von seiner Begegnung mit Michail S. Gorbatschow, und voller Neugier, ob und welche Auswirkungen dessen Politik des gesellschaftlichen Umbaus (Perestrojka) und Transparenz (Glasnost) auf die politische Entwicklung in der DDR haben könnte.

Fast zwei Stunden nahm sich Egon Bahr damals Zeit für mich, hörte mir zu, stellte sehr genaue Fragen und bezog mich in seine Gedanken und Überlegungen ein. Die Zeit verging wie im Flug und ich erinnere mich noch heute an die unglaubliche Intensität dieses Austauschs und an den Umstand, dass Egon Bahr mich in diesem Gespräch auf seine Augenhöhe hob, also in keiner Sekunde die großen Unterschiede zwischen unserer politischen und Lebenserfahrung spüren ließ. Egon Bahr fragte nicht nur, er stellte seine eigenen Ideen auf den Prüfstand, fragte nach meiner Meinung zu seinen damaligen Erkenntnissen. Es war eine seiner beeindruckend großen Gaben, anderen geduldig zuhören zu können, seine eigene Meinung nicht mit der ihm eigenen zweifellos großen Autorität zu verstärken, sondern andere Argumente aufzunehmen, in unnachahmlicher Weise das Wesentliche aus ihnen herauszufiltern, präzise nachzufragen und eigene Ideen logisch schlüssig und praktisch druckreif zu formulieren. Es war der Beginn eines fast drei Jahrzehnte andauernden, zunehmend freundschaftlichen Miteinanders in sehr unterschiedlichen beruflichen und politischen Zusammenhängen und, rückblickend betrachtet, war es ein unerhörtes Privileg, von diesem lebensklugen Menschen lernen zu dürfen und sogar mit ihm zeitweilig an gemeinsamen Projekten zu arbeiten. Ich hatte in den folgenden Jahren nach unserer ersten Begegnung in unregelmäßigem Abstand Gelegenheit ihn erneut zu treffen und zumeist fand er die Zeit auch zu einem bilateralen Austausch. Offiziell trafen wir uns in der zweiten Hälfte der 1980er Jahre ein- bis zweimal im Jahr im Rahmen der zwischen meinem damaligen Institut und dem Institut für Friedensforschung und Sicherheitspolitik an der Universität Hamburg stattfindenden bilateralen Konsultationen.

In ständiger Erinnerung bleibt dabei eine legendäre Sitzung in der Wendezeit. Ursprünglich geplant als bilaterales Arbeitstreffen brachte es die politische Entwicklung in der damaligen DDR mit sich, dass die Vertreterinnen und Vertreter des IPW einen Teil des politischen Spektrums der Teilnehmenden des damaligen Runden Tisches repräsentierten. Egon Bahrs unnachahmliche Verknüpfung von politischer Analyse und konzeptioneller Planung war in dieser Situation beeindruckend. Aus der Hypothese, dass mit der Einführung der Demokratie in der DDR die wichtigste gesellschaftspolitische Legitimation für die Existenz von zwei deutschen Staaten verschwinden würde, schlussfolgerte er scharf, dass es deshalb umso mehr jetzt darauf ankäme, die außenpolitische Legitimation - die damals in der Teilung Deutschlands manifestierte europäische Spaltung - friedlich zu überwinden. Bahr war immer der Auffassung, dass die Schaffung der deutschen Einheit nur in einem europäischen Einigungsprozess aufgefangen werden konnte. Die von ihm entwickelte und unter der Kanzlerschaft von Willy Brandt umgesetzte neue Ostpolitik mit ihrem Kern, dem „Wandel durch Annäherung“, verfolgte dieses Konzept einer Harmonisierung europäischer Einigung und deutscher Annäherung über 25 Jahre hinweg mit großer Konsequenz. Egon Bahr mag sich nicht vorgestellt haben, dass die Regime Osteuropas und auch der DDR binnen kurzer Zeit zusammenbrechen würden, und dass die europäische Einigung Mühe haben würde mit der deutschen Einheit Schritt zu halten. Dabei bleibt unbestritten, dass es ohne die Politik Willy Brandts und Egon Bahrs weder die politischen Umbrüche im östlichen Europa, noch die Erosion der Machtbasis der SED in der DDR gegeben hätte, welche die friedliche Revolution im Wendeherbst 1989 überhaupt erst ermöglichten.

Egon Bahr war wegen seiner Überzeugung, dass Veränderung die Anerkennung des Bestehenden erfordere, zeitlebens und aus unterschiedlichen Gründen oft politischen Anfeindungen ausge- 
setzt. Am meisten aber traf ihn der Vorwurf nach 1989, er hätte die politische Opposition in der DDR zu lange unterschätzt und viel zu lange am Dialog mit den Herrschenden festgehalten. Ersteres hat er in einer späteren persönlichen Rückschau zwar selbst eingeräumt, letzteres aber kann ihm nicht ernsthaft vorgehalten werden. Es sollte nicht vergessen werden, dass zur damaligen Zeit mehr als 360.000 Soldaten der Sowjetunion auf dem Boden der DDR stationiert waren, dass Gorbatschows Öffnungspolitik nicht nur von den meisten Oberen in der DDR für falsch gehalten wurde, sondern sich auch im eigenen Lande der Ruf nach konservativer Restauration regte und dass - und auch dies wird in historischen Rückblenden oft übersehen - auch im Westen, vor allem in Frankreich und Großbritannien, Sorgen hinsichtlich einer Destabilisierung gerade in der Mitte Europas bestanden.

Egon Bahr war zutiefst davon überzeugt, dass durch Anerkennung der Unterschiede gemeinsame Interessen eher identifiziert und Fortschritte in den Beziehungen, im Interesse der von der Teilung betroffenen Menschen, leichter zu erreichen wären. Ohne den von ihm initiierten legendären „Back Channel“ mit Moskau (in Anlehnung an den zwischen ihm und dem damaligen Sicherheitsberater Henry Kissinger ein Jahr zuvor vereinbarten Kanal für informelle Abstimmungen zwischen dem Bundeskanzleramt und dem Weißen Haus) hätte es das Berlin-Abkommen von 1971 nicht gegeben. Auf diesem Kanal war es möglich, auf kurzem Wege direkte Botschaften zu übermitteln, vertrauensvolle Absprachen zu treffen, Ideen ohne Gefahr vorzeitiger Veröffentlichung zu entwickeln. In der Folge bedienten sich auch die Kanzler Schmidt und Kohl der von Bahr geschaffenen direkten Kanäle.

Für den sich in den 1980er Jahren auf vielen Ebenen erst zögerlich, dann schnell, entwickelnden Dialog zwischen beiden deutschen Staaten fand sich als rahmensetzende Rechtfertigung ein Konzept, an dessen Entwicklung erneut Egon Bahr wesentlichen Anteil hatte - das Modell „gemeinsamer Sicherheit“, einer deutschen und europäischen Verantwortungsgemeinschaft oder eines gemeinsamen „Europäischen Hauses“, wie es von Michail Gorbatschow aufgenommen wurde. Dabei ist daran zu erinnern, dass noch Anfang der 1980er Jahre eine neue Eiszeit zwischen Ost und West im Entstehen war, die USA und die Sowjetunion neue nukleare Mittel- und Kurzstreckenraketen auf deutschem Boden stationierten, jedes Plädoyer für eine engere sicherheitspolitische Zusammenarbeit zwischen den beiden deutschen Staaten zwar vernünftig schien, aber auch politische Risiken im Kontext der großen Politik bedeutete. Egon Bahr verstand besser als viele andere die politische Notwendigkeit, sich gerade bei einer drohenden Zuspitzung von Konfrontation besonders, und aller Kritik und Anfeindung zum Trotze, um die sachdienliche Zusammenarbeit mit dem Gegenüber zu bemühen, um nachhaltigen Schaden zu vermeiden und die Bedingungen für einen friedlichen Wandel zu verbessern. Und anders als viele andere nahm er das Risiko auf sich und entwickelte jene Gesprächsformate, welche den Dialog und die Zusammenarbeit auch im politischen und sogar sicherheitspolitischen Raum ermöglichten. Gelegentlich als „Nebenaußenpolitik“ verunglimpft, waren es gerade diese Gesprächsformate bei denen in der Tradition der früheren „Back Channels" nunmehr mit wissenschaftlichem oder Parteienbezug Ideen entwickelt, Botschaften übermittelt und Grundlagen für eine Annäherung der Standpunkte zur Friedenspolitik in der Mitte Europas geschaffen wurden.

\section{Die Entwicklung des deutsch-deutschen wissenschaftlichen Dialoges über Sicherheitspolitik}

Eine geregelte deutsch-deutsche wissenschaftliche Zusammenarbeit zu sicherheitspolitischen Themen gab es bis zum Beginn der 1980er Jahre nicht, und sie wäre, wie sich später noch bestätigen sollte, wegen der besonderen Entstehungsbedingungen der deutschen Teilung auf großes Misstrauen bei den jeweiligen östlichen und westlichen Nachbarn in Europa gestoßen. Die deutsch-deutschen Beziehungen waren insgesamt lange eine ausschließliche Domäne der hohen Politik, und insbesondere in der DDR waren persönliche Kontakte und gar Kooperationen außerhalb staatlicher Kontrolle nach Westdeutschland - zumal nach West-Berlin - unerwünscht. Die internationalen Entwicklungen an der Wende zu den 1980er Jahren brachten jedoch eine neue Lage hervor, welche die deutsch-deutschen Beziehungen stark beeinflussen und auch dem bilateralen wissenschaftlichen Austausch überraschend neue Chancen einräumen sollte.

Anfang des Jahrzehnts hatte sich im europäischen Ost-WestVerhältnis ein neuer Kalter Krieg entwickelt. Durch den sowjetischen Einmarsch in Afghanistan (1979) wurde eine kurze Phase europäischer Entspannung abrupt beendet, die im Jahre 1975 mit der Unterzeichnung der Helsinki-Schlussakte der KSZE ihren Höhepunkt hatte. Die Sowjetunion und die USA hatten danach begonnen, ihre atomaren Arsenale in Europa auf- und nachzurüsten und sie setzten auch in ihrer wechselseitigen politischen Rhetorik zunehmend auf offene Konfrontation. In den beiden deutschen Staaten wurde das fast unaufhaltsam erscheinende Vereisen des Verhältnisses zwischen Moskau und Washington mit wachsender Besorgnis registriert. Dass insbesondere eine militärische Konfrontation in der Mitte Europas Zerstörungen riesigen Ausmaßes heraufbeschwören würde, war angesichts der Konzentration der sich auf deutschem Boden gegenüberstehenden Waffen und Streitkräfte für die Bevölkerung beider Staaten keine Angelegenheit von Spekulation. Es war jedoch nicht allein die Sorge, vor den Folgen eines drohenden Krieges, die auf beiden Seiten der teilenden Grenze das Interesse beförderte, einer wachsenden Verschlechterung des politischen Klimas entgegenzuwirken. Die Ostpolitik Willy Brandts und Egon Bahrs sowie die europäische Entspannungspolitik der 1970er Jahre hatten die Folgen der deutschen Teilung für die Menschen auf beiden Seiten spürbar gelindert. Starke innenpolitische Unterstützung für eine neue Eiszeit in den deutsch-deutschen Beziehungen war unter diesen Vorzeichen in beiden deutschen Staaten nicht zu erwarten. Selbst die Führung der DDR mochte sich nicht den Risiken eines neuen politischen Konfrontationskurses aussetzen, der in weiten Teilen der Bevölkerung - neben der Sorge über drohende Rückschritte im Reiseverkehr - die gewachsene Unzufriedenheit vor allem mit der Versorgungslage in der DDR nur weiter verstärken konnte.

Die SED-Führung hatte dabei auch die wirtschaftlichen und fiskalischen Vorteile einer dauerhaften und engen Kooperation mit der Bundesrepublik im Blick, mit deren Hilfe sich besonders akute Defizite in der Versorgung und der Tilgung von Auslandsschulden kurzfristig beheben ließen. Aber auch in der Bundesrepublik war die DDR als Versorgungslieferantin für Berlin (West) sowie als exklusiver Außenmarkt unter EG-Bedingungen und als eine solvente Schuldnerin wirtschaftlich interessant. Auf beiden Seiten der Grenze wurde indes befürchtet, dass entstandene politische Spielräume, für ein wechselseitig vorteilhaftes 
Miteinander, auf Dauer verschwinden könnten, gerieten die deutsch-deutschen Beziehungen erst in einen ungesteuerten Sog der Ost-West-Konfrontation. So unterschiedlich die Motive und Interessen also in beiden Staaten waren, so stark trafen sich die Interessen in Deutschland Ost und West im Schnittpunkt, die bilateralen Beziehungen möglichst zu konsolidieren und dadurch vor äußeren Einbrüchen abzuschirmen.

Die Erkenntnis, dass vor allem die Deutschen von einer weiteren politischen Zuspitzung und militärischen Konfrontation in Europa Schlimmes zu befürchten hätten, beflügelte das wechselseitige Nachdenken über politische Alternativen zur materiellen und rhetorischen Aufrüstung - allerdings auch den Widerstand jener, die eine enge Zusammenarbeit zwischen beiden Staaten, in welcher Form auch immer, prinzipiell für falsch hielten. So war die Tolerierung von wissenschaftlichen Kontakten, die Beteiligung an - oder die Initiierung von neuen - politiknahen Dialogen (heute würde man sie als Track-2 ${ }^{1}$ bezeichnen) zunächst nicht immer und von allen Beteiligten motiviert, tatsächlich gemeinsame Lösungen für gemeinsame Probleme zu suchen. Das Ausforschen von Wissenschaftlerinnen und Wissenschaftlern ist dabei ebenso aktenkundig wie Versuche gezielter Einflussnahme auf die Gespräche und die daran Beteiligten. ${ }^{2}$

Die Entwicklung der Kontakte und Begegnungen allein auf solche Motive zu beschränken, wäre jedoch rückblickend falsch und irreführend. Ich erinnere mich an seinerzeit durchaus manifeste politische Versuche, den Dialog, gerade wegen seiner dort vorherrschenden Sachlichkeit und Offenheit, zu beeinflussen oder gar zu unterbinden. Tatsächlich boten sich trotz aller Beschränkungen, unter dem Dach eines gemeinsamen Interesses an Stabilität und Zusammenarbeit zwischen den beiden deutschen Staaten, erstaunliche Möglichkeiten für einen problemlösungsorientierten Gedankenaustausch und die Entstehung von sich allmählich festigenden regelmäßigen Gesprächskanälen zur Sicherheitspolitik. Mitunter war dabei die persönliche Autorität und Integrität der Protagonisten dieser Gesprächskanäle, insbesondere eben auch jene Egon Bahrs, hilfreich und auf beiden Seiten schützend.

Der zunächst sehr vorsichtigen Anbahnung wissenschaftlicher Kontakte zwischen den beiden deutschen Staaten kam zugute, dass etwa gleichzeitig neue friedens- und sicherheitspolitische Gesprächszusammenhänge im globalen Maßstab entstanden. Vor allem die von den Vereinten Nationen eingesetzte Unabhängige Kommission für Abrüstung und Sicherheit (PalmeKommission) hatte mit ihrem 1982 unter maßgeblicher Mitwirkung von Egon Bahr veröffentlichten Bericht und Konzept der „Gemeinsamen Sicherheit“3 einen für die deutsch-deutschen Beziehungen geradezu ideal anwendbaren Referenzrahmen für eine Intensivierung friedens- und sicherheitspolitisch motivierter Kooperation geschaffen. Zunächst waren es überwiegend institutionalisierte Dialogplattformen, die zu Anlaufstellen für einen regelmäßigen Gedankenaustausch wurden.

Der Politische Club in Berlin (West), die Evangelische Akademie in Loccum und das Haus Rissen in Hamburg gehörten im Westen dazu, im Osten unter anderem das DDR-Komitee für Europäische Sicherheit und bald dann auch das IPW. Wie bereits angedeutet,

1 Tanja Paffenholz/Melanie Seegräf (2002), Stärkung von Friedensallianzen, Eschborn: GTZ, S. 8.

2 Ausführlich hierzu Hubertus Knabe (1990), Die unterwanderte Republik, Berlin: Propyläen.

3 Der Palme-Bericht. Bericht der Unabhängigen Kommission für Abrüstung und Sicherheit, Berlin: Severin und Siedler 1982. entwickelten sich im sicherheitspolitischen Kontext, neben den wissenschaftlichen Kontakten auch Dialoge zwischen der SED und der SPD, unter anderem über die Einrichtung von atom- und chemiewaffenfreien Zonen auf deutschem Boden, später auch über politikformende oder -begleitende Ideologien. Die direkten Kontakte zwischen den Parteien erleichterten bilaterale Gespräche zunehmend auch auf anderen Ebenen, darunter auch zwischen Forschungseinrichtungen beider deutscher Staaten. Umgekehrt sollten diese Gespräche auch wiederum aus eigener Kraft Transmissionen auf die Ebene der Politik erlauben. Insofern waren diese Gespräche im Grenzbereich zwischen Politik und angewandter Wissenschaft angesiedelt, eine Art „Track 1,5“4 bzw. eben auch eine Art „Back Channel" für einen besonders sensiblen Politikbereich.

Die damalige Führung der DDR verfolgte mit der Billigung von politiknahen Kontakten auf der Ebene regierungsnaher Forschungsinstitute, neben der Sondierung von strategischen Diskursen im politischen Raum, einen weiteren für sie wichtigen Zweck. Die „wissenschaftlichen Dialoge“ boten einen unverfänglichen Raum für direkte Konsultationen und Verabredungen außerhalb des diplomatischen Protokolls zur Vorbereitung offizieller Vereinbarungen. Das Institut für Internationale Politik und Wirtschaft der DDR geriet zwischen 1980 bis 1985 zum vielfrequentierten Anlaufpunkt für Dutzende hochrangige Vertreterinnen und Vertreter aus Politik und Wirtschaft der Bundesrepublik Deutschland. In der Folge dieser Begegnungen öffneten sich zunehmend auch Türen für wissenschaftliche Kooperationen in beiden Richtungen. Viele entwickelten sich im ersten Schritt erst nach dem persönlichen Kennenlernen von Politikerinnen und Politikern beider Seiten, die eigentlich in erster Linie nach legitimierten Wegen für weitere regelmäßige Kommunikationen untereinander suchten. Auch führende Politiker der CDU und CSU machten hiervon ab 1982 regen Gebrauch, zum Beispiel, um nach dem Regierungswechsel innenpolitisch möglichst unauffällig gegenüber der DDR-Führung ihr Interesse an der Fortsetzung der von ihnen vormals heftig kritisierten Entspannungspolitik zu bekunden.

Zwischen 1984 und 1989, nach der Amtsübernahme der Leitungsgeschäfte des IFSH durch Egon Bahr, wurden die wissenschaftlichen Konsultationen regelmäßiger, stärker problembezogen und lösungsorientiert. Insgesamt vier Diskussionsrunden fanden bis 1987 abwechselnd in Berlin und Hamburg statt ${ }^{5}$; später kamen für das IFSH im deutsch-deutschen Kontext noch weitere Begegnungen unter Einbeziehung von hohen Offizieren der NVA und der Bundeswehr hinzu. ${ }^{6}$

\section{Politische Wirkung oder Selbsttäuschung: Versuch einer Bewertung des Sicherheitsdialoges}

Haben die Gespräche zwischen wissenschaftlichen Einrichtungen beider deutscher Staaten über die Verbreitung ihrer Ergebnisse hinaus

4 Die Bezeichnung „Track 1,5“ wurde erst Ende der 1990er in der Mediationsforschung gebräuchlich. Track 1,5 verweist auf das enge Zusammenwirken von Politik und beratender Expertise zur Lösung politischer Probleme. Vgl. u.a. Nan, Susan Allan (2005), Track one-and-Half Diplomacy, in: Ron J. Fisher (Hg.), Paving the Way, Lanham: Lexington Books, S. 161-173.

5 Gemeinsame Sicherheit und Friedliche Koexistenz, Ein gemeinsamer Report des IFSH (Hamburg) und des IPW (Berlin/DDR) über ihre wissenschaftlichen Diskussionen. Hamburger Beiträge zur Friedensforschung und Sicherheitspolitik 27/1988.

6 An den von Egon Bahr 1988 initiierten Gesprächen nahmen u.a. Generale der Militärakademie „Friedrich Engels“ der DDR und der Führungsakademie der Bundeswehr teil. 
eine politische Wirkung gehabt, bzw. eine solche überhaupt erzielen können? War die Annahme eines Erfolgs spezifischer deutschdeutscher Beiträge für den Erhalt des Friedens und der Stabilität in Europa berechtigt oder vor dem Hintergrund der Interessen der Führungsmächte der Bündnisse von Warschauer Vertrag und NATO lediglich selbstberuhigend und in politischer Hinsicht eine Illusion? Wie weit trifft der Vorwurf aus den Reihen der politischen Opposition in der untergehenden DDR zu, die politikbegleitenden wissenschaftlichen Gespräche zwischen Ost und West seien weniger ein Beitrag zum Frieden als eine Krücke zur Verlängerung der SEDHerrschaft gewesen? Haben sich die Teilnehmenden einer Selbsttäuschung hinsichtlich ihrer Einflussmöglichkeiten hingegeben?

Nacheilende Schlaumeierei hat zumeist einfache Antworten auf diese Fragen parat: Eine erkennbare politische Wirkung habe es nicht gegeben; die von den Alliierten gezogenen Grenzen der Souveränität beschränkten die Spielräume eigenständigen Handelns; die Aufwertung der DDR als Gesprächspartnerin in der europäischen Politik habe den Herrschenden zusätzliche Legitimation für ihre Machtausübung im Innern verschafft. Bei genauerer Betrachtung sind diese und ähnliche Antworten aber nur auf den ersten Blick plausibel. Sie sind jedenfalls unvollständig und in verabsolutierter Form historisch unzutreffend.

Allein die Tatsache, dass Anfang der 1980er Jahre politiknahe wissenschaftliche Kontakte zwischen der DDR, der Bundesrepublik und Berlin (West) möglich wurden, war bereits eine Folge eingetretener politischer Wirkungen der europäischen Entspannungspolitik im Rahmen des Helsinki-Prozesses und ihrer Vorläufer, darunter der neuen Ostpolitik der Brandt/Scheel-Regierung an der Schwelle zu den 1970er Jahren. Das von Egon Bahr ersonnene Konzept des „Wandels durch Annäherung“, 7 dem an der Wende zu den 1970er Jahren die neue Ostpolitik Willy Brandts folgte, brachte jene Entspannungspolitik hervor, in der sich die Regierungen der realsozialistischen Staaten um der eigenen innenpolitischen Glaubwürdigkeit willen auf Prinzipien einließen, welche die alltägliche Missachtung von Grund- und Menschenrechten erst zu einer legitimen Projektionsfläche oppositioneller Kritik machten.

In Polen und Ungarn vollzog sich der politische Umschlag früher und gewiss zunächst couragierter als in der DDR und der damaligen Tschechoslowakei, aber auch in diesen beiden unmittelbaren Nachbarstaaten der Bundesrepublik entwickelten sich Erosionsprozesse bis in die politischen und akademischen Führungsschichten hinein, deren rasante Beschleunigung nach Beginn der Öffnungspolitik in der Sowjetunion, vor allem in den Jahren 1988 und 1989, unter anderem eben auch auf die konzeptionellen Debatten zwischen Ost und West über eine gemeinsame Zukunft in Europa zurückzuführen waren. Wer sich im Grundsatz einig ist, hat es schwer, im Alltag anderes zu vertreten.

Bekanntlich hat dabei die Vernunft der Argumente nicht zu einer anderen Politik der SED-Spitze geführt, jedoch die Durchsetzungskraft und Legitimität ihres absoluten Machtanspruchs so stark unterwandert, dass dem Zusammenbruch der immer mehr verkrustenden Herrschaft letztlich kein nennenswerter politischer Widerstand mehr entgegengesetzt werden konnte. Auf die friedenswissenschaftlichen Kontakte gemünzt, bedeutete dies, dass keine überzeugenden konzeptionellen

7 Egon Bahr präsentierte sein berühmtes Konzept erstmals 1963, bei einer Rede an der Evangelischen Akademie in Tutzing. „sozialistischen“ Alternativen zur „Gemeinsamen Sicherheit“ in der DDR mehr mehrheitsfähig waren, die den orthodoxen Teilen der Führungsschicht im Herbst 1989 als ein Fluchtpunkt sicherpolitischen Handelns zur Verfügung gestanden hätten.

Was die oft bezweifelten Spielräume eigenständigen politischen Handelns der Deutschen betrifft, so hatte die Beschränkung der Souveränität infolge des Potsdamer Abkommens von 1945 fraglos begrenzende Auswirkungen. Allerdings bezog sich der Souveränitätsvorbehalt der Alliierten vor allem auf den Status, nicht grundsätzlich auf die von beiden deutschen Staaten verfolgte Politik.

Beide Staaten waren anerkannte Mitglieder der Vereinten Nationen und sie befanden sich in militärischen Bündnissen. Beide waren gleichberechtigte Vertragsstaaten internationaler Rüstungskontrolle und Abrüstung und hatten humanitäre und völkerrechtliche Konventionen ratifiziert. Wie die Bundesrepublik Deutschland verfügte auch die DDR über eigene außenpolitische Spielräume, die von der Führung im eigenen Interesse auch so weit wie möglich genutzt wurden. So führte der 1985 unter Michail S. Gorbatschow eingeschlagene Kurswechsel in der Gesellschafts-, Außen- und Sicherheitspolitik der Sowjetunion die im Westen verbreitete Annahme einer vollständigen Abhängigkeit der DDR von sowjetischer Vormundschaft insofern ad absurdum, als sich die orthodoxe Parteiführung der SED den Ideen von „Glasnost“ und „Perestrojka“ zunehmend offen widersetzte. Anders als in den Jahren zuvor, als die politische Führung unter Erich Honecker die Annäherung in den deutsch-deutschen Beziehungen als ein wohlfeiles eigenes Instrument zur Zügelung konfrontativer Politikkonzeptionen in Moskau erachtete - also auch hier bereits eine eigenständige Politik verfolgte - sah sie nun in der in Aussicht gestellten Annäherung Moskaus an Bonn eine Gefahr für die dauerhafte Fortexistenz der DDR als eigenständigem Staat - und reagierte erneut mit offener Renitenz.

Die mehr oder weniger offizielle wissenschaftliche Entourage der Außen- und Sicherheitspolitik der DDR, die zuvor mit voller Absicht begleitend in den Kurs der Entspannung zwischen Ost und West eingeflochten wurde, mochte diese neuerliche Volte in der Außen- und Sicherheitspolitik der SED-Spitze nicht mehr mitmachen. Zum einen wollte sie ihre neu gewonnenen intellektuellen Freiheiten nicht mehr ohne Weiteres preisgeben, zum anderen griff das konzeptionelle Credo Gorbatschows, ein „neues Denken“ für das Atomzeitalter zu entwickeln, exakt die Überlegungen zur „Gemeinsamen Sicherheit" und zur „Sicherheitspartnerschaft" auf, die sich wichtige Teile der friedens- und sicherheitspolitischen Politikberatung in der DDR inzwischen zu eigen gemacht hatten. Der 1988 gegründete Wissenschaftliche Rat für Friedensforschung diskutierte mit der „Entmilitarisierung von Sicherheit" und der „deutschen Verantwortungsgemeinschaft" Themen, die wenige Jahre zuvor noch als offen „konterrevolutionär“ gegolten hätten - jetzt aber nur noch von einer mächtigen Minderheit denunziert wurden. ${ }^{8}$

Die Gespräche zwischen Ost und West wurden unter den genannten Vorzeichen zunehmend offen, vertrauensvoll und weitgehend frei von taktischer Rücksichtnahme geführt, wodurch sich Gesprächszusammenhänge verdichteten und neue Netzwerke politischer Kommunikation bildeten.

8 Erhard Crome/Lutz Kirschner, Der SED-Reformdiskurs der achtziger Jahre. Zu Deutungen, Kontexten und einem neuen Archiv, in: Initial Berliner Debatte 3/2000, S. 87-102. 
Es mag für die Kolleginnen und Kollegen des IFSH seinerzeit ein Wunder gewesen sein, als im Frühjahr 1990 - anlässlich des letzten Treffens mit dem IPW in Hamburg - die nunmehr auf Seiten des IPW versammelten Repräsentanten unterschiedlicher Parteien und Bürgerbewegungen sehr viel stärker untereinander stritten, als mit ihren westdeutschen Gesprächspartnern. Sie setzten am „Runden Tisch" ${ }^{\prime 9}$ im IFSH-Gebäude in Hamburg-Blankenese fort, was sie für ihre jeweiligen Interessengruppen seit dem Spätherbst 1989 an den „Runden Tischen“ in der DDR praktizierten. So aufregend die Atmosphäre der Debatten damals war, so zutreffend ist auch, dass die widerstreitenden Positionen auf der Seite der DDR-Teilnehmer sich auf Inhalte der vormaligen konzeptionellen Auseinandersetzungen in den Gesprächen zwischen beiden Instituten bezogen, jedoch die Linien der Auffassungsunterschiede nicht mehr zwischen West und Ost verliefen.

Die heutige Erinnerung an konstruktive Debatten und an eine mitunter fast kollegiale Atmosphäre der wissenschaftlichen Begegnungen in den 1980er Jahren mag rückblickend die sicherheitspolitischen Umstände der Ost-West-Konstellation in Europa verklärt erscheinen lassen. Vom Ende der DDR her betrachtet auch aus dem Blickwinkel der totalen moralischen Ablehnung des gescheiterten Versuchs einer sozialistischen deutschen Alternative - wird heute gelegentlich auch verurteilt, was während des Kalten Krieges im Interesse der Deutschen auf beiden Seiten der Grenze unternommen wurde, um die Teilung Deutschlands und Europas zu stabilisieren. Die Lage war jedoch komplexer und kritischer als Geschichtsbücher nachzeichnen können.

Die deutsche Teilung war das Ergebnis des von Deutschland angezettelten Eroberungskrieges. Sie bildete in den Jahrzehnten nach seinem Ende für die meisten europäischen Nachbarn eine nicht verhandelbare Garantie deutscher Zähmung. Für die beiden hegemonialen Großmächte war sie das Fundament bipolarer Stabilität auf dem Kontinent. Mehr als 500.000 Soldaten der Westgruppe der sowjetischen Streitkräfte und Angehörige der Nationalen Volksarmee waren auf dem Boden der DDR stationiert. ${ }^{10}$ Welche Maßnahmen die Sowjetunion im Falle einer politischen und militärischen Krise in der DDR ergriffen hätte, um sich und um die eigenen Soldaten auf deutschem Boden zu schützen, war selbst den Herrschenden in Berlin bis zum Schluss nicht völlig klar. Zumindest vor 1985 hätte jedoch fraglos jeder Versuch eines beförderten politischen Umsturzes in der DDR in eine Katastrophe gemündet. Selbst für die Zeit danach bis zum Frühjahr 1990, kann nicht davon ausgegangen werden, dass der politische Kurswechsel unter Gorbatschow im Krisenfall eine drohende militärische Eskalation unter Einschluss der sowjetischen Truppen verhindert hätte.

Die Truppen der Westgruppe waren in der DDR nicht sonderlich beliebt und eine politisch motivierte Revolte hätte sich früher oder später mit hoher Wahrscheinlichkeit zumindest lokal auch gegen militärische Standorte gerichtet. ${ }^{11}$ Die innenpolitisch schwieriger werdende Lage in der Sowjetunion, der beginnende Zerfall des Warschauer Vertrages und eine mögliche militärische Krise in der DDR hätten auch dem Reformer Gorbatschow

9 Anders als bei dem „Runden Tisch“ in Berlin, der in Wirklichkeit rechteckig war, handelte es sich bei den Gesprächen im IFSH tatsächlich um einen runden Tisch.

10 Wissenschaftlicher Rat für Friedensforschung an der Akademie der Wissenschaften der DDR, Daten, Fakten, Publikationen, Heft I-II/1988.

11 Selbst im "friedlichen Wendeherbst" 1989 gab es einige Protestzwischenfälle an den Kasernentoren der sowjetischen Westgruppe. kaum eine andere Wahl gelassen, als aus eigenem Interesse mit Härte zu reagieren. Der nachgereichte Vorwurf, die Dialoge und Verhandlungen über Sicherheit und Abrüstung zwischen Ost und West hätten das politische Regime der DDR stabilisiert, zeugt insofern von realitätsfernem Urteil.

Umgekehrt ist richtig, dass die gleichzeitigen bilateralen Gespräche und Verhandlungen auf verschiedenen Ebenen in ihrer Gesamtheit erst jenen äußeren stabilen Rahmen in der Mitte Europas schufen, der die Erosion der Machtstruktur in der DDR und das anwachsende Selbstbewusstsein zu zivilem Ungehorsam in den Kirchen und auf den Straßen möglich machten. In der DDR gab es, anders als in Polen, keine gefestigten Strukturen politischer Opposition - jedenfalls nicht vor den Kommunalwahlen im Mai 1989. Wer hätte den politischen Umbruch in der DDR unter Berücksichtigung der großen Verantwortung für den Frieden und die Sicherheit, auch der europäischen Nachbarn, gestalten sollen?

Der frühere Außenminister der DDR, Otto Winzer, hatte weitblickend das von Egon Bahr entwickelte Konzept des „Wandels durch Annäherung“ einst als „Konterrevolution auf Filzlatschen“12 bezeichnet. Das Zerbröseln der Machtstrukturen der SED, die eine demokratische Organisation neben sich nicht dulden wollte, war die Voraussetzung für die friedlichen Revolutionen im Wendeherbst 1989. Die von Egon Bahr wesentlich geprägten Gespräche zwischen den Wissenschaftlerinnen und Wissenschaftlern aus Ost und West über den friedlichen Wandel in Europa und Deutschland hatten daran einen vielleicht nur kleinen, aber wichtigen Anteil.

1991 holte mich Egon Bahr an sein Hamburger Institut. Mit seinen Mitarbeiterinnen und Mitarbeitern entwickelte Egon Bahr seine Vorstellungen für eine Europäische Sicherheitsgemeinschaft weiter, einem System kollektiver Sicherheit für Europa. Sein visionärer politischer Weitblick und sein Fokus auf die konkrete Gestaltung langfristiger politischer Veränderungsprozesse beflügelten. Das Ergebnis gemeinsamer Arbeit, das Konzept einer „Europäischen Sicherheitsgemeinschaft"13, hat bis heute nicht an Aktualität verloren, es liefert gerade für die aktuelle Diskussion über die Zukunft des Friedens in Europa vor dem Hintergrund eines drohenden neuen Kalten Krieges zeitgemäße Antworten.

Bis in sein letztes Lebensjahr hinein hatte ich das Glück, mit Egon Bahr zusammenzuarbeiten. Bis zuletzt trieb ihn die Überzeugung, dass die Rückkehr eines Kalten Krieges in Europa nur durch Kooperation verhindert werden kann. Seine wichtige, kluge und in allen politischen Lagern gehörte Stimme wird in diesem Bemühen fehlen.

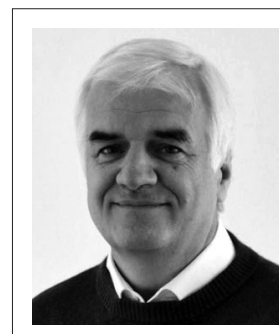

Hans-Joachim Giessmann (Prof. Dr. Dr.) ist Executive Director der Berghof Foundation und Mitherausgeber von Sicherheit und Frieden $-\mathrm{S}+\mathrm{F}$.

12 Nach Auskunft des damaligen Leiters der Grundsatzabteilung des DDRAußenministeriums, Siegfried Bock. Vgl. Interview mit der "Jungen Welt" vom 30./31. Juli 2005.

13 Institut für Friedensforschung und Sicherheitspolitik an der Universität Hamburg: Die Europäische Sicherheitsgemeinschaft: das Sicherheitsmodell für das 21. Jahrhundert, Bonn: SEF, 1995. 